\title{
Bell Inequalities for Graph States
}

\author{
Otfried Gühne, ${ }^{1}$ Géza Tóth, ${ }^{2}$ Philipp Hyllus, ${ }^{3,4}$ and Hans J. Briegel ${ }^{1,5}$ \\ ${ }^{1}$ Institut für Quantenoptik und Quanteninformation, \\ Österreichische Akademie der Wissenschaften, A-6020 Innsbruck, Austria, \\ ${ }^{2}$ Max-Planck-Institut für Quantenoptik, Hans-Kopfermann-Straße 1, D-85748 Garching, Germany, \\ ${ }^{3}$ Institut für Theoretische Physik, Universität Hannover, Appelstraße 2, D-30167 Hannover, Germany, \\ ${ }^{4}$ Institute for Mathematical Sciences, Imperial College London, 48 Prince's Gardens, SW7 London 2AZ, UK, \\ ${ }^{5}$ Institut für Theoretische Physik, Universität Innsbruck, Technikerstraße 25, A-6020 Innsbruck, Austria
}

(Dated: September 7, 2018)

\begin{abstract}
We investigate the non-local properties of graph states. To this aim, we derive a family of Bell inequalities which require three measurement settings for each party and are maximally violated by graph states. In turn, for each graph state there is an inequality maximally violated only by that state. We show that for certain types of graph states the violation of these inequalities increases exponentially with the number of qubits. We also discuss connections to other entanglement properties such as the positivity of the partial transpose or the geometric measure of entanglement.
\end{abstract}

PACS numbers: 03.65.Ud, 03.67.-a, 03.67.Lx, 03.67.Pp

Quantum theory predicts correlations which are stronger than the correlations of local hidden variable (LHV) models. By definition, LHV models have to obey the constraints of realism and locality: Any observable has a predetermined value, regardless of whether it is measured or not, and the choice of which observable to measure on one party of a multipartite system does not affect the results of the other parties. These constraints lead to the so-called Bell inequalities which put bounds on the correlations. These inequalities turn out to be violated by certain quantum mechanical states 1, 2].

In this letter we address the question, whether graph states allow a LHV description or not. Graph states form a family of multi-qubit states which comprises many popular states such as the Greenberger-Horne-Zeilinger (GHZ) states and the cluster states 3]. Graph states are also crucial for applications: All codewords in the standard quantum error correcting codes correspond to graph states [4] and one-way quantum computation uses graph states as resources 5]. Recently, graph states have been produced in optical lattices [6] and the basic elements of one-way quantum computing have been demonstrated experimentally [7]. Also, general methods for the generation of graph states have been explored [8].

It is a natural and important question whether these tasks and experiments, including the effects of noise can be described by LHV models. To answer this question, we derive a class of Bell inequalities. Each graph state violates one of these inequalities in the GHZ sense, i.e., by saturating all correlation terms and for certain types of graph states the violation of local realism increases exponentially with the number of qubits. In this way we show that tasks like measurement based quantum computing and quantum error correction are far from the realm of LHV theories. Note that the non-locality of special examples of graph states has been shown recently [2].

Graph states are defined as follows. Let $G$ be a

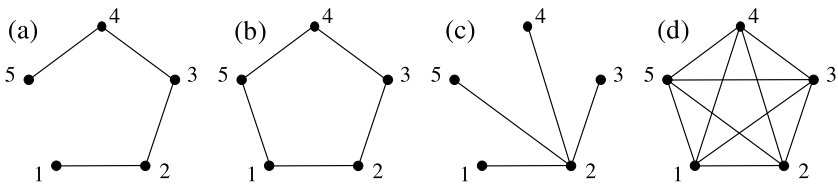

FIG. 1: Types of graphs for the case of five vertices: (a) The linear cluster graph $L C_{5}$. (b) The ring cluster graph $R C_{5}$. (c) The star (or GHZ) graph $S T_{5}$. This describes a GHZ state. (d) The fully connected graph $F C_{5}$. This graph can be obtained from $S T_{5}$ by local complementation on the second qubit (see Lemma 4). It also describes a GHZ state [3].

graph, i.e., a set of $n$ vertices and some edges connecting them. Some interesting graphs are shown in Fig. 1. For each vertex $i$ the neighborhood $N(i)$ denotes the vertices which are connected with $i$. We can associate to each vertex $i$ a stabilizing operator $g_{i}$ by

$$
g_{i}=X^{(i)} \bigotimes_{j \in N(i)} Z^{(j)} .
$$

From now on, $X^{(i)}, Y^{(i)}, Z^{(i)}$ denote the Pauli matrices $\sigma_{x}, \sigma_{y}, \sigma_{z}$, acting on the $i$-th qubit. For instance, for the fully connected three vertex graph, the stabilizing operators are $g_{1}=X^{(1)} Z^{(2)} Z^{(3)}, g_{2}=Z^{(1)} X^{(2)} Z^{(3)}$ and $g_{3}=Z^{(1)} Z^{(2)} X^{(3)}$. The graph state $|G\rangle$ associated with the graph $G$ is the unique $n$-qubit state fulfilling

$$
g_{i}|G\rangle=|G\rangle, \text { for } i=1, \ldots, n .
$$

Physically, the graph describes the perfect correlations in the state $|G\rangle$, since $\left\langle g_{i}\right\rangle=\left\langle X^{(i)} \bigotimes_{j \in N(i)} Z^{(j)}\right\rangle=1$. At the same time, it denotes a possible interaction history leading to $|G\rangle$, i.e., $|G\rangle$ can be produced by an Ising type interaction acting between the connected qubits.

Given the stabilizing operators $g_{i}$, we can look at the group of their products, the so-called stabilizer 11],

$$
S(G)=\left\{s_{j}, j=1, \ldots, 2^{n}\right\} ; \quad s_{j}=\prod_{i \in I_{j}(G)} g_{i}
$$


where $I_{j}(G)$ denotes a subset of the vertices of $G$. If a certain generator $g_{k}$ appears in the product in Eq. (3), (i.e., $k \in I_{j}(G)$ ), we say that $s_{j}$ contains $g_{k}$. The group $S(G)$ is commutative and has $2^{n}$ elements. Of course, for all these elements $s_{j}|G\rangle=|G\rangle$ holds. A last property we have to mention is the fact that

$$
\sum_{i=1}^{2^{n}} s_{i}=2^{n}|G\rangle\langle G|
$$

holds, as can be checked by direct calculation [3].

Now we present our idea for the derivation of Bell inequalities. Given a graph $G$ all the stabilizing operators are of the form

$$
s_{i}(G)=\bigotimes_{k=1}^{n} O_{i}^{(k)},
$$

where the single qubit observables are either the identity or one of the Pauli matrices: $O_{i}^{(k)} \in$ $\left\{\mathbb{1}, \pm X^{(k)}, \pm Y^{(k)}, \pm Z^{(k)}\right\}$. We look at the operator

$$
\mathcal{B}(G)=\sum_{i}^{2^{n}} s_{i}(G)=\sum_{i}^{2^{n}} \bigotimes_{k=1}^{n} O_{i}^{(k)}
$$

To give a simple example, this operator for the fully connected graph state for three qubits reads

$$
\begin{aligned}
\mathcal{B}\left(F C_{3}\right) & =\mathbb{1}^{(1)} \mathbb{1}^{(2)} \mathbb{1}^{(3)}+X^{(1)} Z^{(2)} Z^{(3)}+Z^{(1)} X^{(2)} Z^{(3)} \\
& +Z^{(1)} Z^{(2)} X^{(3)}+Y^{(1)} Y^{(2)} \mathbb{1}^{(3)}+Y^{(1)} \mathbb{1}^{(2)} Y^{(3)} \\
& +\mathbb{1}^{(1)} Y^{(2)} Y^{(3)}-X^{(1)} X^{(2)} X^{(3)}
\end{aligned}
$$

We take this $\mathcal{B}$ as the Bell operator and compute a bound

$$
\mathcal{C}(G) \equiv \mathcal{C}(\mathcal{B})=\max _{\text {LHV }}|\langle\mathcal{B}\rangle|,
$$

where the maximum of the absolute value of the mean value $\langle\mathcal{B}\rangle$ is taken over all LHV models. Here, it suffices to look at deterministic LHV models which have to assign definite values $\{+1,-1\}$ to the observables $O_{k}^{(i)}$, whenever $O_{k}^{(i)} \neq \mathbb{1}[\underline{9}$. This is due to the fact that nondeterministic LHV models can be viewed as deterministic LHV models where the hidden variables are not known. In principle, in the definition of $\mathcal{B}$ the Pauli matrices can be replaced by arbitrary dichotomic observables. Since we are interested in graph states, we will, however, always use $X, Y, Z$.

If we can find for a given graph $G$ a bound $\mathcal{C}(G)<2^{n}$, the nonlocality of the graph state $|G\rangle$ is detected. This is due to Eq. (4), which implies that for the graph state $\langle\mathcal{B}\rangle=2^{n}$ holds. Also, the graph state violates the Bell inequality maximally. In the example of Eq. (7) we will see later that $\mathcal{C}\left(F C_{3}\right)=6$. This gives rise to the Bell inequality $\left|\left\langle\mathcal{B}\left(F C_{3}\right)\right\rangle\right| \leq 6$ which is violated by the state $\left|F C_{3}\right\rangle$. In the following, it will also be useful to compare the strength of the Bell inequalities by the normalized parameter $\mathcal{D}(G):=\mathcal{C}(G) / 2^{n}$ or $1 / \mathcal{D}$. Note that we have a valid Bell inequality whenever $\mathcal{D}<1$.

So the main task is to find the value of $\mathcal{C}(G)$ or $\mathcal{D}(G)$. An exact calculation is, in general, very demanding. However, as we will show, it is quite easy to obtain bounds on $\mathcal{D}(G)$ depending on the structure of the graph $G$, especially when we can identify some subgraphs where the bounds are already known. Together with the exact calculation of $\mathcal{D}(G)$ for graphs with a small number of qubits this allows us to derive some general results for arbitrary graphs. Let us first note two useful facts about the dependence of $\mathcal{D}$ on the LHV models.

Lemma 1. We can restrict our attention to LHV models which assign +1 to all $Z$ measurements.

Proof. In an element $s_{j}$ of the stabilizer we have $O^{(i)} \in$ $\left\{Y^{(i)}, Z^{(i)}\right\}$ iff the number of $Y$ and $X$ in $N(i)$ is odd. So if a LHV model assigns -1 to $Z^{(i)}$, we can, by changing the signs for $Z^{(i)}, Y^{(i)}$ and for all $X^{(k)}$ and all $Y^{(k)}$ with $k \in N(i)$, obtain a LHV model with the same mean value of $\mathcal{B}$ and the desired property.

Lemma 2. Let $\mathcal{B}$ be a Bell operator for an arbitrary graph and let $\mathcal{B}^{\prime}$ be the Bell operator which is obtained from $\mathcal{B}$ by making a permutation

$$
P:\left\{\mathbb{1}^{(i)}, X^{(i)}, Y^{(i)}, Z^{(i)}\right\} \rightarrow\left\{\mathbb{1}^{(i)}, X^{(i)}, Y^{(i)}, Z^{(i)}\right\}
$$

of the observables on one qubit. Then $\mathcal{D}(\mathcal{B})=\mathcal{D}\left(\mathcal{B}^{\prime}\right)$.

Proof. It suffices to show the Lemma for a transposition $O^{(i)} \leftrightarrow \tilde{O}^{(i)}$. Some transpositions are a renaming of variables, and the interesting transpositions are of the type $A^{(i)} \leftrightarrow \mathbb{1}^{(i)}$ with $A^{(i)}=X^{(i)}, Y^{(i)}, Z^{(i)}$, say $A^{(i)}=X^{(i)}$ for definiteness. If a given LHV model $L H V_{1}$ assigns +1 to $X^{(i)}$ the transposition $X^{(i)} \leftrightarrow \mathbb{1}^{(i)}$ does not change $\langle\mathcal{B}\rangle$. If the LHV model assigns -1 to $X^{(i)}$ we can construct a new LHV model $L H V_{2}$ from $L H V_{1}$ by flipping the signs from $Y^{(i)}, Z^{(i)}$. This fulfills $\left\langle\mathcal{B}^{\prime}\right\rangle_{L H V_{1}}=-\langle\mathcal{B}\rangle_{L H V_{2}}$. This proves the claim, since $\mathcal{C}$ is defined via the absolute value.

Now we derive an estimate for $\mathcal{D}(G)$, when $G$ is built out of two other graphs $G_{1}$ and $G_{2}$ in a certain way.

Lemma 3. Let $G_{1}, G_{2}$ be two graphs and let $G$ be the graph which comprises $G_{1}$ and $G_{2}$ and one single connection between one vertex of $G_{1}$ and one of $G_{2}$, i.e., $G=G_{1}-G_{2}$. Then

$$
\mathcal{D}(G) \leq \mathcal{D}\left(G_{1}\right) \mathcal{D}\left(G_{2}\right) .
$$

Proof. The proof is given in the Appendix.

It is much more demanding to derive bounds on $\mathcal{D}(G)$ when $G$ is made out of subgraphs in a more complicated way than the way above. However, it is easy to see that $\mathcal{D}(G)<1$ whenever $G$ contains a subgraph $G_{1}$ with $\mathcal{D}\left(G_{1}\right)<1$. This is due to the fact that the stabilizer of $G_{1}$ is a subset of the stabilizer of $G$ up to some extra $Z$ terms which can be neglected due to Lemma 1 .

Finally, we want to show the invariance of $\mathcal{D}$ under the so-called local complementation of a graph. This 
TABLE I: Value for $\mathcal{D}(G)$ for different interesting graphs (see Fig. 1) and different numbers of qubits $n$.

\begin{tabular}{|c||c|c|c|c|c|c|c|c|}
\hline$n$ & 3 & 4 & 5 & 6 & 7 & 8 & 9 & 10 \\
\hline \hline$L C_{n}$ & $3 / 4$ & $3 / 4$ & $5 / 8$ & $9 / 16$ & $8 / 16$ & $7 / 16$ & $25 / 64$ & $22 / 64$ \\
\hline$R C_{n}$ & $3 / 4$ & $3 / 4$ & $5 / 8$ & $7 / 16$ & $7 / 16$ & $6 / 16$ & $21 / 64$ & $19 / 64$ \\
\hline$S T_{n}$ & $3 / 4$ & $3 / 4$ & $5 / 8$ & $10 / 16$ & $9 / 16$ & $9 / 16$ & $34 / 64$ & $34 / 64$ \\
\hline$F C_{n}$ & $3 / 4$ & $3 / 4$ & $5 / 8$ & $10 / 16$ & $9 / 16$ & $9 / 16$ & $34 / 64$ & $34 / 64$ \\
\hline
\end{tabular}

transformation acts as follows: One picks up a vertex $i_{0}$ and inverts the neighborhood $N\left(i_{0}\right)$, i.e., all connections between two vertices belonging to $N\left(i_{0}\right)$ are cut and vertices in $N\left(i_{0}\right)$ which were unconnected become connected. Connections between $N\left(i_{0}\right)$ and the rest of the graph are not affected. To give an example the graph $S T_{n}$ can be transformed by a local complementation on the central qubit into the graph $F C_{n}$ (see Fig. 1). The local complementation of a graph acts on the graph state as a local unitary transformation of the (local) Clifford group [3, 10]. This means that it transforms on each qubit Pauli matrices into Pauli matrices. So we have:

Lemma 4. Let $G_{1}$ be a graph and $G_{2}$ be a graph which arises from $G_{1}$ by local complementation. Then $\mathcal{D}\left(G_{1}\right)=\mathcal{D}\left(G_{2}\right)$.

Proof. Since the local complementation maps Pauli matrices to Pauli matrices on each qubit, $\mathcal{D}$ is not changed due to Lemma 2.

After showing in the previous Lemmata how to estimate $\mathcal{D}(G)$ when the values of subgraphs are given, it is now time to calculate $\mathcal{D}(G)$ for graphs with a small number of qubits $n$. This can easily be done by a computer, by checking $\langle\mathcal{B}\rangle$ for all the $8^{n}$ LHV models. In these calculations, Lemma 1 reduces the computational complexity significantly. The numerical results for interesting graphs up to ten qubits are shown in Table I.

Let us shortly discuss these results. First, note that all of the states in the table violate a Bell inequality of the type $|\langle\mathcal{B}\rangle| \leq \mathcal{C}(G)$ since for all states and $n$ in Table I $\mathcal{D}(G)<1$ holds. Then, it is remarkable, that long linear chains and large rings have a small $\mathcal{D}$ (i.e. the violation of the Bell inequality is large), while the violation for the GHZ state is not so large. This in contrast to the usual Mermin inequality for several qubits. Due to Lemma 4, the values $\mathcal{D}\left(S T_{n}\right)$ and $\mathcal{D}\left(F C_{n}\right)$ always coincide. However, it is interesting that $\mathcal{D}\left(F C_{6}\right) \not \leq \mathcal{D}\left(F C_{3}\right) \mathcal{D}\left(F C_{3}\right)$, thus a generalization of Lemma 3 to arbitrary connections between the graphs is not true. In general, we have:

Theorem 1. Any graph state violates local realism. Proof. If the graph consists only of two connected vertices, the graph state is equivalent to a two qubit singlet state, which violates the original Bell inequality [1]. Connected graphs with more vertices always contain a subgraph with three vertices. Due to Table 1 and the argumentation after Lemma 3 this implies that the graph state violates local realism.
Theorem 2. Let $G_{1}, G_{2}, G_{3}, \ldots$ be a family of tree graphs (i.e., graphs which do not contain any closed ring) with an increasing number of vertices such that each $G_{i}$ contains as a subgraph a linear chain of a size which increases linearly with $i$. Then violation of a Bell inequality for $\left|G_{i}\right\rangle$ increases exponentially with $i$.

Proof. From Lemma 3 and Table I it follows that for linear cluster graphs $L C_{i}$ the value $1 / \mathcal{D}\left(L C_{i}\right)$ increases exponentially. For tree graphs Lemma 3 can be applied again to show that $\mathcal{D}\left(G_{i}\right)$ is smaller than the $\mathcal{D}$ from the longest linear cluster in $G_{i}$.

Similar statements as the one expressed in Theorem 2 can be derived for other families of graphs. In fact, all graphs which are built by connecting an increasing number of subgraphs via single edges exhibit an exponentially increasing violation of local realism, due to Lemma 3.

Let us compare the Bell inequalities with other entanglement properties. Here, our restriction to the observables $X, Y, Z$ becomes crucial. A Bell inequality $|\langle\mathcal{B}\rangle| \leq \mathcal{C}(G)$ is equivalent to a witness of the type

$$
\mathcal{W}=\mathcal{D}(G)-|G\rangle\langle G|,
$$

i.e., a quantum mechanical state violates the Bell inequality iff $\langle\mathcal{W}\rangle<0$ [9]. This has two consequences. First, since all fully separable states $|\psi\rangle=$ $|a\rangle|b\rangle \ldots|n\rangle$ admit a LHV description, here $\mathcal{D}(G) \geq$ $\max _{|a\rangle|b\rangle \ldots|n\rangle}|\langle a, b, \ldots, n \mid G\rangle|^{2}$ has to hold. The quantity $1-\max _{|a\rangle|b\rangle \ldots|n\rangle}|\langle a, b, \ldots, n \mid G\rangle|^{2}$ has been shown to be an entanglement monotone for multipartite systems, the so-called geometric measure of entanglement [12]. So our bounds on $\mathcal{C}$ also deliver lower bounds for this measure of entanglement for graph states. In turn, the fact that the geometric measure equals $1 / 2$ for all GHZ states implies that always $\mathcal{D}\left(S T_{n}\right) \geq 1 / 2$. Second, we can state:

Theorem 3. If $\mathcal{D}(G) \geq 1 / 2$ then the Bell inequality $|\langle\mathcal{B}\rangle| \leq \mathcal{C}(G)$ detects only states which have a negative partial transpose with respect to each partition.

Proof. Let us fix a bipartite splitting for the multipartite system. The graph state has a Schmidt decomposition $|G\rangle=\sum_{i=1}^{k} a_{i}|i i\rangle$ with respect to this splitting. It is known that then $1 / k \leq a_{0}^{2} \leq 1 / 2$ if $a_{0}$ is the biggest Schmidt coefficient 13]. If we define $|\psi\rangle=\sum_{i=1}^{k}|i i\rangle / \sqrt{k}$ it is also known that the witness $\tilde{\mathcal{W}}=\mathbb{1} / k-|\psi\rangle\langle\psi|$ detects only states which have a non-positive partial transpose with respect to this partition [14]. However the witness $\mathcal{W}$ detects even less states, since $\mathcal{W}-k a_{0}{ }^{2} \tilde{\mathcal{W}}=$ $\left(\mathcal{D}-a_{0}^{2}\right) \mathbb{1}-|G\rangle\left\langle G\left|+k a_{0}^{2}\right| \psi\right\rangle\left\langle\psi\left|\geq k a_{0}^{2}\right| \psi\right\rangle\langle\psi|-| G\rangle\langle G| \geq 0$ which implies that $\langle\mathcal{W}\rangle<0$ only if $\langle\tilde{\mathcal{W}}\rangle<0$.

In conclusion, we have derived a family of Bell inequalities for multipartite systems based on the graph state formalism. These inequalities are maximally violated by graph states and allow to detect the non-locality of all graph states. Also, the inequalities can be related to other topics as the geometric measure of entanglement and the criterion of the partial transposition. The fact 
that graph states do not admit a LHV model strongly suggests that tasks like measurement based quantum computation and quantum error correction cannot be described within classical physics.

We thank Marc Hein for discussions and P.H. thanks the Universität Innsbruck for hospitality. This work has been supported by the DFG (Schwerpunkt Quanteninformationsverarbeitung) and the EU (MEIF-CT-2003500183, IST-2001-38877, -39227, OLAQI).

Appendix - Here we give the proof of Lemma 3. Let us consider a given LHV model and assume that the connection is between the vertices $i_{0} \in G_{1}$ and $j_{0} \in G_{2}$. We can write any stabilizing operator of $G$ as $s_{i j}(G)=a_{i} b_{j}$ where $a_{i}$ contains only $g_{k}$ with $k \in G_{1}$ and $b_{j}$ contains only $g_{k}$ belonging to $G_{2}$. We can arrange the set $\left\{a_{i}\right\}=\mathfrak{a}_{1} \cup \ldots \cup \mathfrak{a}_{6}$ into six disjoint subsets in the following way: $\mathfrak{a}_{1}, \mathfrak{a}_{2}$ contain the $a_{i}$ where $g_{i_{0}}$ is absent, $\mathfrak{a}_{3}, \mathfrak{a}_{4}$ have an $X^{\left(i_{0}\right)}$ at the vertex $i_{0}$ and $\mathfrak{a}_{5}, \mathfrak{a}_{6}$ have a $Y^{\left(i_{0}\right)}$. Note that this implies that $\mathfrak{a}_{3}, \mathfrak{a}_{4}, \mathfrak{a}_{5}$, and $\mathfrak{a}_{6}$ comprise the $a_{i}$ which contain $g_{i_{0}}$. For $a_{i} \in \mathfrak{a}_{1}, \mathfrak{a}_{3}, \mathfrak{a}_{5}$ the LHV model gives $\left\langle a_{i}\right\rangle=+1$ while for the $a_{i} \in \mathfrak{a}_{2}, \mathfrak{a}_{4}$ or $\mathfrak{a}_{6}$ we have $\left\langle a_{i}\right\rangle=-1$. Let us denote the number of elements in the sets $\mathfrak{a}_{1}, \mathfrak{a}_{2}, \mathfrak{a}_{3}, \mathfrak{a}_{4}, \mathfrak{a}_{5}$ and $\mathfrak{a}_{6}$ by $p_{a}, q_{a}, r_{a}, s_{a}, t_{a}$ and $u_{a}$. We can define $\gamma_{a}=: p_{a}-q_{a}, \delta_{a}:=r_{a}-s_{a}$ and $\varepsilon_{a}=t_{a}-u_{a}$. Then we have $\left|\left\langle\mathcal{B}\left(G_{1}\right)\right\rangle\right|=\left|\gamma_{a}+\delta_{a}+\varepsilon_{a}\right| \leq \mathcal{C}\left(G_{1}\right)$. We can do the same ordering for the $b_{j}$, introducing $\mathfrak{b}_{1}, \ldots, \mathfrak{b}_{6}$ and $p_{b}, \ldots, u_{b}$ and $\gamma_{b}, \delta_{b}, \varepsilon_{b}$, with $\left|\gamma_{b}+\delta_{b}+\varepsilon_{b}\right| \leq \mathcal{C}\left(G_{2}\right)$.

Let us investigate the relationships between $\gamma, \delta$, and $\varepsilon$ in some more detail. By flipping the sign which is assigned to $X^{\left(i_{0}\right)}$ by the given LHV model we can construct a new LHV model with $\left|\left\langle\mathcal{B}\left(G_{1}\right)\right\rangle\right|=\left|\gamma_{a}-\delta_{a}+\varepsilon_{a}\right| \leq$ $\mathcal{C}\left(G_{1}\right)$. We can also flip the signs of $Y^{\left(i_{0}\right)}, X^{\left(j_{0}\right)}$ or $Y^{\left(j_{0}\right)}$ leading to new bounds of the type $|\gamma \pm \delta \pm \varepsilon| \leq \mathcal{C}$. All in all, this leads to the conclusion, that for all sixteen combinations of signs

$$
\left|\left(\gamma_{a} \pm \delta_{a} \pm \varepsilon_{a}\right)\left(\gamma_{b} \pm \delta_{b} \pm \varepsilon_{b}\right)\right| \leq \mathcal{C}\left(G_{1}\right) \mathcal{C}\left(G_{2}\right)
$$

holds. Finally, note that the operators $s_{i j}$ can be grouped into 36 groups according to $\mathfrak{s}_{i j}=\mathfrak{a}_{i} \mathfrak{b}_{j}$. The mean values $\left\langle s_{i j}\right\rangle=\left\langle a_{i} b_{j}\right\rangle$ would factorize if there were no connection between the graphs. In this case, the Lemma is trivial.

What changes for the $\left\langle s_{i j}\right\rangle$ due to the extra connection? The $\left\langle s_{i j}\right\rangle$ can be written in a $6 \times 6$ block matrix according to the grouping into the $\mathfrak{s}_{i j}$, where each block bears the sign of the corresponding $\left\langle s_{i j}\right\rangle$. In the blocks $\mathfrak{s}_{i j}$ with $i \leq 2$ or $j \leq 2$ the extra connection only introduces transformations of the type $Z \leftrightarrow \mathbb{1}$ at $i_{0}$ or $j_{0}$, which can be neglected due to Lemma 1. More interesting is the $4 \times 4$ block matrix of the blocks $\mathfrak{s}_{i j}$ with $3 \leq i, j \leq 6$. One can calculate that here the extra connection induces the transformation $\left\{X^{\left(i_{0}\right)} X^{\left(j_{0}\right)}, X^{\left(i_{0}\right)} Y^{\left(j_{0}\right)}, Y^{\left(i_{0}\right)} X^{\left(j_{0}\right)}, Y^{\left(i_{0}\right)} Y^{\left(j_{0}\right)}\right\} \mapsto$ $\left\{Y^{\left(i_{0}\right)} Y^{\left(j_{0}\right)},-Y^{\left(i_{0}\right)} X^{\left(j_{0}\right)},-X^{\left(i_{0}\right)} Y^{\left(j_{0}\right)}, X^{\left(i_{0}\right)} X^{\left(j_{0}\right)}\right\}$ on the vertices $i_{0}$ and $j_{0}$. So, depending on the LHV model, this results in this block matrix in two possible changes of signs. They can be written in the following way:

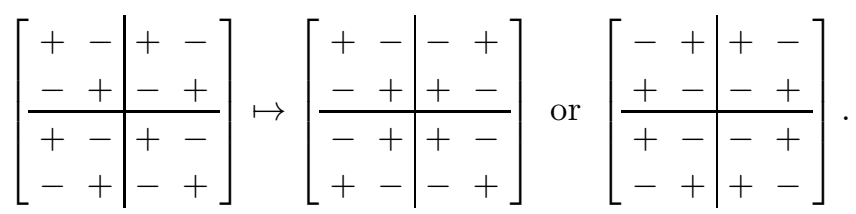

For the first possible transformation we have to show that $\left|\sum_{i j}\left\langle s_{i j}\right\rangle\right|=\mid \gamma_{a}\left(\gamma_{b}+\delta_{b}+\varepsilon_{b}\right)+\delta_{a}\left(\gamma_{b}+\delta_{b}-\varepsilon_{b}\right)+$ $\varepsilon_{a}\left(\gamma_{b}-\delta_{b}+\varepsilon_{b}\right) \mid \leq \mathcal{C}\left(G_{1}\right) \mathcal{C}\left(G_{2}\right)$ This can be derived from Eq. (12), distinguishing 64 cases depending on the signs of $\gamma_{a}, \gamma_{b}, \delta_{a}, \delta_{b}, \varepsilon_{a}$ and $\varepsilon_{b}$. For instance, if $\gamma_{a}, \delta_{a}, \delta_{b} \geq 0$ and $\gamma_{b}, \varepsilon_{a}, \varepsilon_{b}<0$ we use $\left(\gamma_{a}+\delta_{a}+\varepsilon_{a}\right)\left(\gamma_{b}+\delta_{b}+\varepsilon_{b}\right) \leq$ $\gamma_{a}\left(\gamma_{b}+\delta_{b}+\varepsilon_{b}\right)+\delta_{a}\left(\gamma_{b}+\delta_{b}-\varepsilon_{b}\right)+\varepsilon_{a}\left(\gamma_{b}-\delta_{b}+\varepsilon_{b}\right) \equiv$ $\sum_{i j}\left\langle s_{i j}\right\rangle \leq-\left(\gamma_{a}+\delta_{a}-\varepsilon_{a}\right)\left(\gamma_{b}-\delta_{b}+\varepsilon_{b}\right)$, yielding an upper and a lower bound for $\sum_{i j}\left\langle s_{i j}\right\rangle$. The proof of the other 63 cases and the second transformation is similar.

[1] J.S. Bell, Physics 1, 195 (1964); for multipartite Bell inequalities see G. Svetlichny, Phys. Rev. D 35, 3066 (1987); N.D. Mermin, Phys. Rev. Lett. 65, 1838 (1990); D.M. Greenberger et al., Am. J. Phys. 58, 1131 (1990); M. Ardehali, Phys. Rev. A 46, 5375 (1992); S. Popescu and D. Rohrlich, Phys. Lett. A 166, 293 (1992); A.V. Belinskii and D.N. Klyshko, Usp. Fiz. Nauk 163(8) 1 (1993); N. Gisin and H. Bechmann-Pasquinucci, Phys. Lett. A 246, 1 (1998); A. Peres, Found. Phys. 29, 589 (1999); R.F. Werner and M.M. Wolf, Phys. Rev. A 64, 032112 (2001); D. Collins et al., Phys. Rev. Lett. 88, 170405 (2002); M. Żukowski and Č. Brukner ibid. 88, 210401 (2002); W. Laskowski et al., ibid. 93, 200401 (2004).

[2] V. Scarani et al., Phys. Rev. A 71, 042325 (2005), see also D.P. DiVincenzo and A. Peres, ibid. 55, 4089 (1997).

[3] M. Hein, J. Eisert, and H.J. Briegel, Phys. Rev. A 69, 062311 (2004); W. Dür et al., Phys. Rev. Lett. 91, 107903 (2003).

[4] D. Schlingemann and R.F. Werner, Phys. Rev. A 65, 012308 (2002); M. Grassl, A. Klappenecker, and M. Rötteler, in Proc. 2002 IEEE International Symposium on Information Theory, Lausanne, Switzerland, p. 45; K. Chen, H.-K. Lo, quant-ph/0404133

[5] R. Raussendorf and H.J. Briegel, Phys. Rev. Lett. 86, 5188 (2001); M. Nielsen, ibid., 93, 040503 (2004).

[6] O. Mandel et al., Nature (London) 425, 937 (2003).

[7] P. Walther et al., Nature (London) 434, 169 (2005).

[8] D.E. Browne and T. Rudolph, Phys. Rev. Lett. 95, 010501 (2005); A.-N. Zhang et al., quant-ph/0501036

[9] Note that in quantum theory always $\langle\mathcal{B}\rangle \geq 0$, since $\mathcal{B}(G)=2^{n}|G\rangle\langle G|$ is positive. However, defining $\mathcal{C}$ via the absolute value turns out to be convenient later.

[10] M. Van den Nest, J. Dehaene, and B. De Moor, Phys. Rev. A 69, 022316 (2004).

[11] D. Gottesman, Phys. Rev. A 54, 1862 (1996).

[12] T. Wei and P. Goldbart, Phys. Rev. A 68, 042307 (2003).

[13] G. Tóth and O. Gühne, Phys. Rev. Lett. 94, 060501 (2005); see also quant-ph/0501020

[14] A. Sanpera, D. Bruß, and M. Lewenstein, Phys. Rev. A 63, 050301 (2001). 